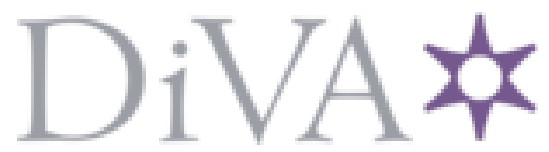

http://www.diva-portal.org

This is [Version unknown!] version of a paper presented at The 36th Chinese Control Conference.

Citation for the original published paper:

Yi, X., Yang, T., Wu, J., Johansson, K H. (2017)

Event-Triggered Control for Multi-Agent Systems with Output Saturation.

In: IEEE

N.B. When citing this work, cite the original published paper.

Permanent link to this version:

http://urn.kb.se/resolve?urn=urn:nbn:se:kth:diva-205566 


\title{
Event-Triggered Control for Multi-Agent Systems with Output Saturation
}

\author{
Xinlei $\mathrm{Yi}^{1}$, Tao Yang ${ }^{2}$, Junfeng $\mathrm{Wu}^{1}$, Karl H. Johansson ${ }^{1}$ \\ 1. The ACCESS Linnaeus Centre, Electrical Engineering, KTH Royal Institute of Technology, 100 44, Stockholm, Sweden \\ E-mail: $\{$ xinleiy, junfengw, kallej\}@kth.se \\ 2. The Department of Electrical Engineering, University of North Texas, Denton, TX 76203 USA \\ E-mail: Tao.Yang@unt.edu
}

\begin{abstract}
We propose distributed static and dynamic event-triggered control laws to solve the consensus problem for multiagent systems with output saturation. Under the condition that the underlying graph is undirected and connected, we show that consensus is achieved under both event-triggered control laws if and only if the average of the initial states is within the saturation level. Numerical simulations are provided to illustrate the effectiveness of the theoretical results and to show that the control laws lead to reduced need for inter-agent communications.
\end{abstract}

Key Words: Consensus, Event-triggered control, Multi-agent systems, Output saturation

\section{Introduction}

Due to its wide applicability, consensus in multi-agent systems has been widely investigated. The basic method is to use a distributed consensus protocol. Specifically, each agent updates its state based on its own and the states of its neighbors in such a way that the final states of all agents converge to a common value, e.g., [1-4]. However, real systems are subject to physical constraints such as input, output, digital communication channels, and sensors constraints. These constraints lead to nonlinearity in the closed-loop dynamics. Thus the behavior of each agent is affected and special attention to these constraints needs to be taken in order to understand their influence on the convergence properties. Here we list some representative examples of such constraints. For example, [5] studies global consensus for discrete-time multi-agent systems with input saturation constraint; [6] considers the leader-following consensus problem for multi-agent systems subject to input saturation; [7] and $[8]$ investigate necessary and sufficient initial conditions for achieving consensus in the presence of output saturation.

In the aforementioned work, the agent updates its state based on the continuous communication with its neighboring agents. However, it may be impractical to require continuous communication in physical applications, as agents can be equipped with embedded microprocessors with limited resources to transmit and collect information. Event-triggered control was introduced partially to tackle this problem [912]. The control in event-triggered control signal is often piecewise constant between the triggering times. The triggering times are determined implicitly by the event conditions to ensure the stability of the closed-loop system. Many researchers studied event-triggered control for multi-agent systems recently, e.g., [13-22]. The key points in eventtriggered control for multi-agent systems are how to design the event-triggered control law and the threshold to determine the corresponding triggering times and how to exclude Zeno behavior. For continuous-time multi-agent systems,

This work is supported by the Knut and Alice Wallenberg Foundation, the Swedish Foundation for Strategic Research, and the Swedish Research Council.
Zeno behavior is that there are infinite number of triggers in a finite time interval [23]. Another important point is how to realize the event-triggered controller in a distributed way since on the one hand for the centralized controller, it normally requires a large amount of agents take action in a synchronous manner and it is not efficient, on the other hand for some decentralized controller, it requires a priori knowledge of some global network parameters, for example, in [15] the smallest positive eigenvalue of the Laplacian matrix needs to be known in advance.

In this paper, we first consider consensus for static eventtriggered control of multi-agent systems with output saturation. Then, we study dynamic event-triggered control law, which is a multi-agent extension of an event-triggered mechanism proposed in [24]. The contributions of this paper are two-fold: 1) we show that consensus is achieved under both static and dynamic event-triggered control laws, which are distributed in the sense that they do not require any a priori knowledge of global network parameters; and 2) we show that the dynamic event-triggered control law is free from Zeno behavior.

The rest of this paper is organized as follows. Section 2 introduces the preliminaries and the problem formulation. The main results are stated in Section 3. Simulations are given in Section 4. Finally, the paper is concluded in Section 5.

Notations: $\|\cdot\|$ represents the Euclidean norm for vectors or the induced 2-norm for matrices. $\mathbf{1}_{n}$ denotes the column vector with each component being 1 and dimension $n$.

\section{Preliminaries}

In this section, we present some definitions from algebraic graph theory [25] and the formulation of the problem.

\subsection{Algebraic Graph Theory}

Let $\mathcal{G}=(\mathcal{V}, \mathcal{E}, A)$ denote a (weighted) undirected graph with the set of agents (vertices or nodes) $\mathcal{V}=\{1, \ldots, n\}$, the set of links (edges) $\mathcal{E} \subseteq \mathcal{V} \times \mathcal{V}$, and the (weighted) adjacency matrix $A=A^{\top}=\left(a_{i j}\right)$ with nonnegative elements $a_{i j}$. A link $(i, j) \in \mathcal{E}$ if $a_{i j}>0$, i.e., if agent $i$ and $j$ can communicate with each other. It is assumed that $a_{i i}=0$ for all $i \in \mathcal{V}$. Let $\mathcal{N}_{i}=\left\{j \in \mathcal{V} \mid a_{i j}>0\right\}$ and 
$\operatorname{deg}_{i}=\sum_{j=1}^{n} a_{i j}$ denotes the neighbors and (weighted) degree of agent $i$, respectively. The degree matrix of graph $\mathcal{G}$ is $D=\operatorname{diag}\left(\left[\operatorname{deg}_{1}, \cdots, \operatorname{deg}_{n}\right]\right)$. The Laplacian matrix is $L=$ $\left(L_{i j}\right)=D-A$. A path of length $k$ between agent $i$ and agent $j$ is a subgraph with distinct agents $i_{0}=i, \ldots, i_{k}=j \in \mathcal{V}$ and edges $\left(i_{j}, i_{j+1}\right) \in \mathcal{E}, j=0, \ldots, k-1$. An undirected graph is connected if there exists at least one path between any two distinct agents.

For connected graphs we have the following well known result [25].

Lemma 1 If an undirected graph $\mathcal{G}$ is connected, then its Laplacian matrix L has a simple eigenvalue at zero with corresponding eigenvector $\mathbf{1}_{n}$ and all other eigenvalues are real and strictly positive.

\subsection{Problem Formulation}

We consider a set of $n$ agents that are modeled as a single integrator with output saturation:

$$
\left\{\begin{array}{l}
\dot{x}_{i}(t)=u_{i}(t) \\
y_{i}(t)=\operatorname{sat}_{h}\left(x_{i}(t)\right)
\end{array} \quad, i \in \mathcal{V}, t \geq 0\right.
$$

where $x_{i}(t) \in \mathbb{R}$ is the state, $u_{i}(t) \in \mathbb{R}$ is the control input, and $y_{i}(t)$ is the measured output. The saturation function sat $_{h}: \mathbb{R} \rightarrow \mathbb{R}$ is defined as

$$
\operatorname{sat}_{h}(s)= \begin{cases}h, & \text { if } s \geq h \\ s, & \text { if }|s|<h \\ -h, & \text { if } s \leq-h\end{cases}
$$

where $h$ is a positive constant, and the interval $[-h, h]$ is referred to as the saturation level. For simplicity, for vector

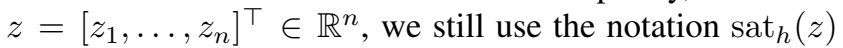
and $\operatorname{define}_{\operatorname{sat}_{h}}(z)=\left[\operatorname{sat}_{h}\left(z_{1}\right), \ldots, \operatorname{sat}_{h}\left(z_{n}\right)\right]^{\top}$.

Remark 1 For the ease of presentation, we study the case where all the agents have the same saturation level and scalar states. However, the analysis in this paper can be easily extended the cases where the agents have different saturation levels and have vector-valued states.

In the literature, the following distributed consensus protocol is often considered, e.g., [7],

$$
u_{i}(t)=-\sum_{j=1}^{n} L_{i j} y_{j}(t)
$$

To implement (3), continuous-time outputs from all neighbours are needed. However, it is often unpractical to require continuous communication in physical applications. Moreover, if at some time $t_{0},\left|x_{i}\left(t_{0}\right)\right|>h$, then due to the continuity of $x_{i}(t)$, there exists $t_{1}>t_{0}$ such that $\left|x_{i}(t)\right| \geq$ $h, \forall t \in\left[t_{0}, t_{1}\right]$. Then $y_{i}(t)=\operatorname{sat}_{h}\left(x_{i}(t)\right)$ is a constant during $\left[t_{0}, t_{1}\right]$. So it is also waste to continuously transmit $y_{i}(t)$ during $\left[t_{0}, t_{1}\right]$ since no new information is provided.

Inspired by the idea of event-triggered control for multiagent systems [13], instead of (3) we use the following eventtriggered control

$$
u_{i}(t)=-\sum_{j=1}^{n} L_{i j} y_{j}\left(t_{k_{j}(t)}^{j}\right)=-\sum_{j=1}^{n} L_{i j} \operatorname{sat}_{h}\left(x_{j}\left(t_{k_{j}(t)}^{j}\right)\right),
$$

where $k_{j}(t)=\operatorname{argmax}_{k}\left\{t_{k}^{j} \leq t\right\}$ with the increasing $\left\{t_{k}^{j}\right\}_{k=1}^{\infty}, j \in \mathcal{V}$ to be determined later. We assume $t_{1}^{j}=$ $0, j \in \mathcal{V}$. Note that the control protocol (4) only updates at the triggering times and is constant between consecutive triggering times.

For simplicity, let $x(t)=\left[x_{1}(t), \ldots, x_{n}(t)\right]^{\top}$, $\hat{x}_{i}(t)=x_{i}\left(t_{k_{i}(t)}^{i}\right), \quad \hat{x}(t)=\left[\hat{x}_{1}(t), \ldots, \hat{x}_{n}(t)\right]^{\top}$, $e_{i}(t)=\operatorname{sat}_{h}\left(\hat{x}_{i}(t)\right)-\operatorname{sat}_{h}\left(x_{i}(t)\right)$, and $e(t)=$ $\left[e_{1}(t), \cdots, e_{n}(t)\right]^{\top}=\operatorname{sat}_{h}(\hat{x}(t))-\operatorname{sat}_{h}(x(t))$.

\section{Event-Triggered Control Law}

In this section, we propose the static and dynamic eventtriggered control laws to determine the triggering time sequence and we show that consensus is achieved under both event-trigger control laws.

\subsection{Static Event-Triggered Control Law}

We first give some lemmas which will be used later.

Lemma 2 Consider the multi-agent system (1) with the event-triggered control protocol (4). The average of all agents' states $\bar{x}(t)=\frac{1}{n} \sum_{i=1}^{n} x_{i}(t)$ is a constant, i.e., $\bar{x}(t)=\bar{x}(0)$ for all $t \geq 0$.

Proof: It follows from (1) and (4) that the time derivative of the average value is given by

$$
\begin{aligned}
\dot{\bar{x}}(t) & =\frac{1}{n} \sum_{i=1}^{n} \dot{x}_{i}(t)=-\frac{1}{n} \sum_{i=1}^{n} \sum_{j=1}^{n} L_{i j} \operatorname{sat}_{h}\left(x_{j}\left(t_{k_{j}(t)}^{j}\right)\right) \\
& =-\frac{1}{n} \sum_{j=1}^{n} \operatorname{sat}_{h}\left(x_{j}\left(t_{k_{j}(t)}^{j}\right)\right) \sum_{i=1}^{n} L_{i j}=0 .
\end{aligned}
$$

Lemma 3 (Lemma 3.2 in [7].) For any constants $a, b$ with $|a| \leq h$,

$$
\int_{a}^{b}\left[\operatorname{sat}_{h}(s)-a\right] d s \geq 0
$$

and the equality holds if and only if $a=b$ or $b \geq a=h$ or $b \leq a=-h$.

Assume that $|\bar{x}(0)| \leq h$. Consider the Lyapunov candidate

$$
V(x)=\sum_{i=1}^{n} \int_{\bar{x}(0)}^{x_{i}}\left[\operatorname{sat}_{h}(s)-\bar{x}(0)\right] d s .
$$

From Lemma 3, we know that $V(t) \geq 0$ due that $|\bar{x}(0)| \leq h$. The derivative of $V(x)$ along the trajectories of system (1) with the event-triggered control (4) is

$$
\begin{aligned}
\dot{V}(x) & =\sum_{i=1}^{n}\left[\operatorname{sat}_{h}\left(x_{i}(t)\right)-\bar{x}(0)\right] \dot{x}_{i}(t) \\
& =\sum_{i=1}^{n} \operatorname{sat}_{h}\left(x_{i}(t)\right) \dot{x}_{i}(t)-\sum_{i=1}^{n} \bar{x}(0) \dot{x}_{i}(t) \\
& =\sum_{i=1}^{n} \operatorname{sat}_{h}\left(x_{i}(t)\right) \dot{x}_{i}(t)+\sum_{i=1}^{n} \bar{x}(0) \sum_{j=1}^{n} L_{i j} \operatorname{sat}_{h}\left(\hat{x}_{j}(t)\right) \\
& =\sum_{i=1}^{n} \operatorname{sat}_{h}\left(x_{i}(t)\right) \dot{x}_{i}(t)+\sum_{j=1}^{n} \bar{x}(0) \operatorname{sat}_{h}\left(\hat{x}_{j}(t)\right) \sum_{i=1}^{n} L_{i j} \\
& =\sum_{i=1}^{n}\left[\operatorname{sat}_{h}\left(\hat{x}_{i}(t)\right)-e_{i}(t)\right] \sum_{j=1}^{n}-L_{i j} \operatorname{sat}_{h}\left(\hat{x}_{j}(t)\right)
\end{aligned}
$$




$$
\begin{aligned}
= & -\sum_{i=1}^{n} \operatorname{sat}_{h}\left(\hat{x}_{i}(t)\right) \sum_{j=1}^{n} L_{i j} \operatorname{sat}_{h}\left(\hat{x}_{j}(t)\right) \\
& +\sum_{i=1}^{n} e_{i}(t) \sum_{j=1}^{n} L_{i j} \operatorname{sat}_{h}\left(\hat{x}_{j}(t)\right) \\
= & \sum_{i=1}^{n}-q_{i}(t) \\
& +\sum_{i=1}^{n} \sum_{j=1, j \neq i}^{n} L_{i j} e_{i}(t)\left[\operatorname{sat}_{h}\left(\hat{x}_{j}(t)\right)-\operatorname{sat}_{h}\left(\hat{x}_{i}(t)\right)\right] \\
\leq & \sum_{i=1}^{n}-q_{i}(t)-\sum_{i=1}^{n} \sum_{j=1, j \neq i}^{n} L_{i j} e_{i}^{2}(t) \\
& -\sum_{i=1}^{n} \sum_{j=1, j \neq i}^{n} L_{i j} \frac{1}{4}\left[\operatorname{sat}_{h}\left(\hat{x}_{j}(t)\right)-\operatorname{sat}_{h}\left(\hat{x}_{i}(t)\right)\right]^{2} \\
= & \sum_{i=1}^{n}-\frac{1}{2} q_{i}(t)+\sum_{i=1}^{n} L_{i i} e_{i}^{2}(t),
\end{aligned}
$$

where

$$
q_{i}(t)=-\frac{1}{2} \sum_{j=1}^{n} L_{i j}\left[\operatorname{sat}_{h}\left(\hat{x}_{j}(t)\right)-\operatorname{sat}_{h}\left(\hat{x}_{i}(t)\right)\right]^{2} \geq 0,
$$

and the last two equalities hold since

$$
\begin{aligned}
\sum_{i=1}^{n} q_{i}(t) & =-\sum_{i=1}^{n} \frac{1}{2} \sum_{j=1}^{n} L_{i j}\left[\operatorname{sat}_{h}\left(\hat{x}_{j}(t)\right)-\operatorname{sat}_{h}\left(\hat{x}_{i}(t)\right)\right]^{2} \\
& =\sum_{i=1}^{n} \sum_{j=1}^{n} \operatorname{sat}_{h}\left(\hat{x}_{i}(t)\right) L_{i j} \operatorname{sat}_{h}\left(\hat{x}_{j}(t)\right) \\
& =\left[\operatorname{sat}_{h}(\hat{x}(t))\right]^{\top} L \operatorname{sat}_{h}(\hat{x}(t)),
\end{aligned}
$$

and the inequality holds because $a b \leq a^{2}+\frac{1}{4} b^{2}$ for all $a, b \in$ $\mathbb{R}$.

Our first main result follows from the above discussion.

Theorem 1 Consider the multi-agent system (1) with the even-triggered control protocol (4). Suppose that the underlying graph $\mathcal{G}$ is undirected and connected. Suppose $x(0) \neq \bar{x}(0) \mathbf{1}_{n}$. Given a constant $\sigma_{i} \in(0,1)$ and the first triggering time $t_{1}^{i}=0$, every agent $i$ determines its triggering time sequence $\left\{t_{k}^{i}\right\}_{k=2}^{\infty}$ by

$$
t_{k+1}^{i}=\max _{r \geq t_{k}^{i}}\left\{r: e_{i}^{2}(t) \leq \frac{\sigma_{i}}{2 L_{i i}} q_{i}(t), \forall t \in\left[t_{k}^{i}, r\right]\right\},
$$

with $q_{i}(t)$ defined in (7). Then consensus is achieved if and only if

$$
|\bar{x}(0)| \leq h .
$$

Proof: (Necessity) This part of the proof is a special case of the proof of Theorem 3.1 in [7] with some minor modifications. We thus omit the proof here.

(Sufficiency) If $|\bar{x}(0)| \leq h$, then from Lemma 3 we know that $V(t) \geq 0$ and $V(t)=0$ if and only if $x_{i}(t)=\bar{x}(0), i \in$
$\mathcal{V}$. From (6) and (8), we have

$$
\begin{aligned}
& \dot{V}(x) \leq \sum_{i=1}^{n}-\frac{1}{2} q_{i}(t)+\sum_{i=1}^{n} L_{i i} e_{i}^{2}(t) \\
& \leq \frac{1}{2}\left(1-\sigma_{\text {max }}\right) \sum_{i=1}^{n}-q_{i}(t) \\
& =-\frac{1}{2}\left(1-\sigma_{\max }\right)\left[\operatorname{sat}_{h}(\hat{x}(t))\right]^{\top} L \operatorname{sat}_{h}(\hat{x}(t)) \leq 0,
\end{aligned}
$$

where $\sigma_{\max }=\max \left\{\sigma_{1}, \ldots, \sigma_{n}\right\}<1$. Note that

$$
\begin{aligned}
& {\left[\operatorname{sat}_{h}(x(t))\right]^{\top} L \operatorname{sat}_{h}(x(t)) } \\
= & {\left[\operatorname{sat}_{h}(\hat{x}(t))+e(t)\right]^{\top} L\left[\operatorname{sat}_{h}(\hat{x}(t))+e(t)\right] } \\
\leq & 2\left[\operatorname{sat}_{h}(\hat{x}(t))\right]^{\top} L \operatorname{sat}_{h}(\hat{x}(t))+2 e^{\top}(t) L e(t) \\
\leq & 2\left[\operatorname{sat}_{h}(\hat{x}(t))\right]^{\top} L \operatorname{sat}_{h}(\hat{x}(t))+2\|L\|\|e(t)\|^{2} \\
\leq & 2\left[\operatorname{sat}_{h}(\hat{x}(t))\right]^{\top} L \operatorname{sat}_{h}(\hat{x}(t))+\frac{\|L\| \sigma_{\max }}{\min _{i} L_{i i}} \sum_{i=1}^{n} q_{i}(t) \\
= & \left(2+\frac{\|L\| \sigma_{\max }}{\min _{i} L_{i i}}\right)\left[\operatorname{sat}_{h}(\hat{x}(t))\right]^{\top} L \operatorname{sat}_{h}(\hat{x}(t)),
\end{aligned}
$$

where the first inequality holds since $L$ is positive semidefinite and $[a+b]^{\top} L[a+b] \leq 2 a^{\top} L a+2 b^{\top} L b, \forall a, b \in$ $\mathbb{R}^{n}$, the second inequality holds because $a^{\top} L a \leq$ $\|L\|\|a\|^{2}, \forall a \in \mathbb{R}^{n}$, and the last inequality holds due to (8). Then we have

$$
\begin{aligned}
\dot{V}(x) & \leq-\frac{\left(1-\sigma_{\max }\right) \min _{i} L_{i i}}{4 \min _{i} L_{i i}+2\|L\| \sigma_{\max }}\left[\operatorname{sat}_{h}(x(t))\right]^{\top} L \operatorname{sat}_{h}(x(t)) \\
& \leq 0 .
\end{aligned}
$$

Moreover we have $\dot{V}(t)=0$ if and only if $\operatorname{sat}_{h}(x(t))=$ $a \mathbf{1}_{n}$ for some $a \in \mathbb{R}$. This is equivalent to $x(t)=a \mathbf{1}_{n}$ for some $a \in \mathbb{R}$, otherwise $x(t) \geq h \mathbf{1}_{n}$ and $x(t) \neq h \mathbf{1}_{n}$ or $x(t) \leq-h \mathbf{1}_{n}$ and $x(t) \neq-h \mathbf{1}_{n}$, however, both cases contradict $|\bar{x}(t)| \leq h$. Then by LaSalle Invariance Principle [26] $\lim _{t \rightarrow+\infty} x_{i}(t)=\bar{x}(0)$, for all $i \in \mathcal{V}$.

Remark 2 The control law (8) is referred to as a static static triggering law since it depends only on the current state of the considered agent. The event-triggered control law is distributed since each agent's control action only depends on its neighbours' state information, without any prior knowledge of global parameters, such as the eigenvalue of the Laplacian matrix.

The event-triggered control law reduces the overall communication among agents. It is essential to exclude Zeno behavior [23]. However, we do not know whether Zeno behavior can be excluded or not in the above event-triggered control law. In order to exclude Zeno behavior, in the next section, we propose a dynamic event-triggered control law.

\subsection{Dynamic Event-triggered Control Law}

Inspired by [24], we introduce an internal dynamic variable $\eta_{i}$ to agent $i$ :

$$
\dot{\eta}_{i}(t)=-\beta_{i} \eta_{i}(t)+\frac{\sigma_{i}}{2} q_{i}(t)-L_{i i} e_{i}^{2}(t),
$$

with $\eta_{i}(0)>0, \beta_{i}>0$ and $\sigma_{i} \in(0,1)$. This dynamic variable is correlated in the event-triggered rule, as defined in our second main result. 
Theorem 2 Consider the multi-agent system (1) with the even-triggered control protocol (4). Suppose that the underlying graph $\mathcal{G}$ is undirected and connected. Suppose $x(0) \neq \bar{x}(0) \mathbf{1}_{n}$. Given a constant $\theta_{i}>0$ and the first triggering time $t_{1}^{i}=0$, every agent $i$ determines its triggering time sequence $\left\{t_{k}^{i}\right\}_{k=2}^{\infty}$ by

$$
\begin{array}{r}
t_{k+1}^{i}=\max _{r \geq t_{k}^{i}}\left\{r: \theta_{i}\left(L_{i i} e_{i}^{2}(t)-\frac{\sigma_{i}}{2} q_{i}(t)\right) \leq \eta_{i}(t),\right. \\
\left.\forall t \in\left[t_{k}^{i}, r\right]\right\},
\end{array}
$$

with $q_{i}(t)$ defined in (7) and $\eta_{i}(t)$ defined in (11). Then (i) there is no Zeno behavior; (ii) consensus is achieved if and only if

$$
|\bar{x}(0)| \leq h .
$$

Proof: (i) From equation (11) and condition (12), we have

$$
\dot{\eta}_{i}(t) \geq-\beta_{i} \eta_{i}(t)-\frac{1}{\theta_{i}} \eta_{i}(t) .
$$

Thus

$$
\eta_{i}(t) \geq \eta_{i}(0) e^{-\left(\beta_{i}+\frac{1}{\theta_{i}}\right) t}>0 .
$$

Next, we show that there is no Zeno behavior by contradiction. Suppose that there exists Zeno behavior, then there exists an agent $i$, such that $\lim _{k \rightarrow \infty} t_{k}^{i}=T_{0}$, where $T_{0}$ is a positive constant. Let $\varepsilon_{0}=\frac{1}{4 \sqrt{\theta_{i} L_{i i}^{3}} h} e^{-\frac{1}{2}\left(\beta_{i}+\frac{1}{\theta_{i}}\right) T_{0}}>0$. Then, there exists a positive integer $N\left(\varepsilon_{0}\right)$ such that

$$
t_{k}^{i} \in\left[T_{0}-\varepsilon_{0}, T_{0}\right], \forall k \geq N\left(\varepsilon_{0}\right) .
$$

Since $\left|\operatorname{sat}_{h}(s)\right| \leq h$, we have $\left|u_{i}(t)\right| \leq 2 h L_{i i}$. From $q_{i}(t) \geq 0$ and $\left|\operatorname{sat}_{h}\left(s_{1}\right)-\operatorname{sat}_{h}\left(s_{2}\right)\right| \leq\left|s_{1}-s_{2}\right|$, we conclude that one sufficient condition to guarantee the inequality in condition (12) is

$$
\left|\hat{x}_{i}(t)-x_{i}(t)\right| \leq \frac{1}{\sqrt{\theta_{i} L_{i i}}} e^{-\frac{1}{2}\left(\beta_{i}+\frac{1}{\theta_{i}}\right) t} .
$$

Again from $\left|\dot{x}_{i}(t)\right|=\left|u_{i}(t)\right| \leq 2 h L_{i i}$ and $\mid \hat{x}_{i}\left(t_{k}^{i}\right)-$ $x_{i}\left(t_{k}^{i}\right) \mid=0$ for any triggering time $t_{k}^{i}$, we conclude that one sufficient condition to inequality (14) is

$$
\left(t-t_{k}^{i}\right) 2 h L_{i i} \leq \frac{1}{\sqrt{\theta_{i} L_{i i}}} e^{-\frac{1}{2}\left(\beta_{i}+\frac{1}{\theta_{i}}\right) t} .
$$

Then

$$
\begin{aligned}
& t_{N\left(\varepsilon_{0}\right)+1}^{i}-t_{N\left(\varepsilon_{0}\right)}^{i} \geq \frac{1}{2 \sqrt{\theta_{i} L_{i i}^{3}} h} e^{-\frac{1}{2}\left(\beta_{i}+\frac{1}{\theta_{i}}\right) t_{N\left(\varepsilon_{0}\right)+1}^{i}} \\
& \geq \frac{1}{2 \sqrt{\theta_{i} L_{i i}^{3}} h} e^{-\frac{1}{2}\left(\beta_{i}+\frac{1}{\theta_{i}}\right) T_{0}}=2 \varepsilon_{0}
\end{aligned}
$$

which contradicts (13). Therefore, there is no Zeno behavior.

(ii) (Necessity) This part of the proof is a special case of the proof of Theorem 3.1 in [7] with some minor modifications. We thus omit the proof here.

(Sufficiency) Let $\eta(t)=\left[\eta_{1}(t), \ldots, \eta_{n}(t)\right]^{\top}$. Consider the Lyapunov candidate

$$
W(x, \eta)=V(x)+\sum_{i=1}^{n} \eta_{i},
$$

where $V(t)$ is given (5). If $|\bar{x}(0)| \leq h$, then it follows from Lemma 3 that $V(x) \geq 0$ and $V(x)=0$ if and only if $x_{i}=\bar{x}(0), i=1, \ldots, n$. The derivative of $W(x, \eta)$ along the trajectories of system (1) and system (11) with the eventtriggered control protocol (4) is

$$
\begin{gathered}
\dot{W}(x, \eta)=\dot{V}(x)+\sum_{i=1}^{n} \dot{\eta}_{i}(t) \\
\leq \frac{1}{2}\left(1-\sigma_{\max }\right) \sum_{i=1}^{n}-q_{i}(t)-\sum_{i=1}^{n} \beta_{i} \eta_{i}(t) \leq 0 .
\end{gathered}
$$

Then by LaSalle Invariance Principle [26], we have

$$
\lim _{t \rightarrow \infty} x_{i}(t)=\bar{x}(0), i \in \mathcal{V} \text {. }
$$

Remark 3 The static triggering law (8) can be seen as a limit case of the dynamic triggering law (12) when $\theta_{i}$ grows large.

\section{Simulations}

In this section, a numerical example is provided to demonstrate the theoretical results. We choose $h=1$ in the saturation function. Consider a connected network of four agents with the Laplacian matrix

$$
L=\left[\begin{array}{rrrr}
5.7 & -2.2 & 0 & -3.5 \\
-2.2 & 7.9 & -5.7 & 0 \\
0 & -5.7 & 6.7 & -1 \\
-3.5 & 0 & -1 & 4.5
\end{array}\right]
$$

whose topology is shown in Fig. 1. The initial value of each agent is randomly selected within the interval $[-5,5]$. We first choose $x(0)=[2.513,-2.449,0.060,1.991]^{\top}$, the average initial state is $\bar{x}(0)=0.5288$ and the condition $|\bar{x}(0)| \leq h$ is thus satisfied. Therefore, according to Theorems 1 and 2, consensus is achieved. Fig. 2 (a) shows the state evolution under the static triggering law (8) with $\sigma_{i}=0.9$. Fig. 2 (b) shows the corresponding triggering times for each agent. Fig. 3 (a) shows the state evolution under the dynamic triggering law (12) with $\sigma_{i}=0.9$, $\eta_{i}(0)=10, \beta_{i}=1$ and $\theta_{i}=1$. Fig. 3 (b) shows the corresponding triggering times for each agent. It can be seen that 1) under both event-triggered laws consensus is achieved; 2) comparing with the static triggering law (8), the dynamic triggering law (12) determines less triggering times for each agent; 3) the trajectories under the static triggering law (8) are more smooth than the trajectories under the dynamic triggering law (12).

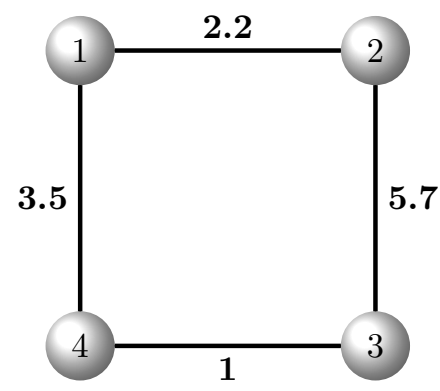

Fig. 1: The communication topology. 


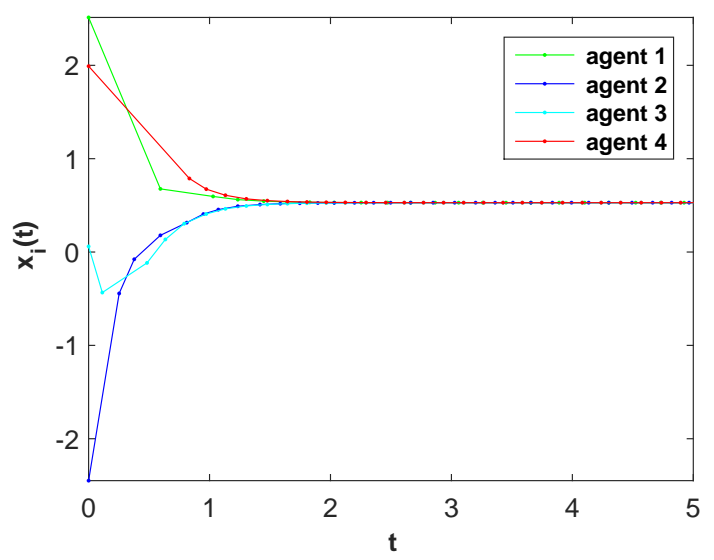

(a)

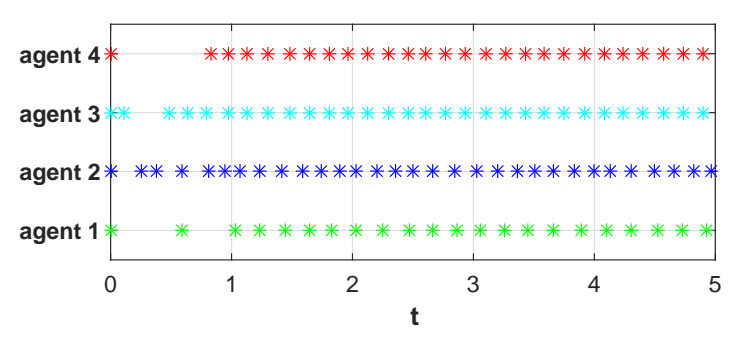

(b)

Fig. 2: (a) The state evolution under the static triggering law (8). (b) The triggering times for each agent under the static triggering law (8).

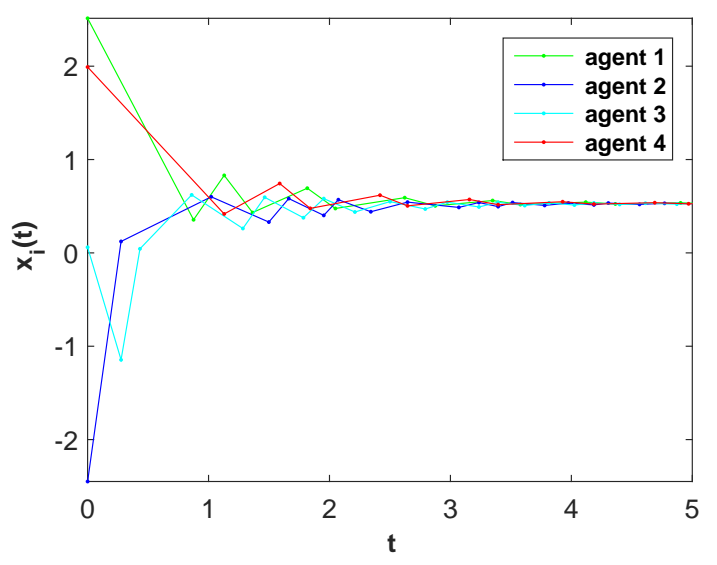

(a)

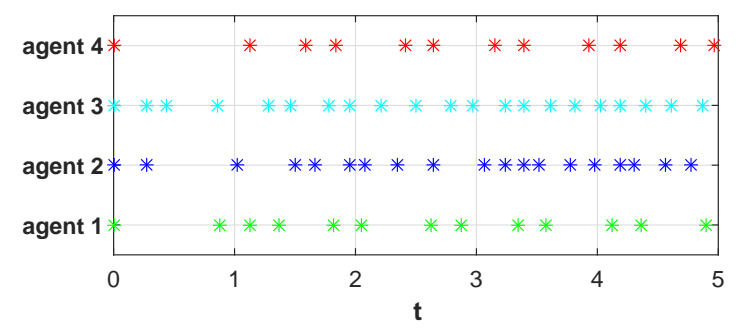

(b)

Fig. 3: (a) The state evolution under the dynamic triggering law (12). (b) The triggering times for each agent under the dynamic triggering law (12).
We next choose the initial value as $x(0)=$ $[2.513,0.551,0.060,1.991]^{\top}$. The average initial state is $\bar{x}(0)=1.2788$, so the condition $|\bar{x}(0)| \leq h$ is not satisfied in this case. Therefore, according to Theorems 1 and 2, consensus is not achieved. Fig. 4 shows the state evolution under the static triggering law (8) with $\sigma_{i}=0.9$. Fig. 5 shows the state evolution under the dynamic triggering law (12) with $\sigma_{i}=0.9, \eta_{i}(0)=10, \beta_{i}=1$ and $\theta_{i}=1$. It can be seen that under both event-triggered laws consensus is not achieved in this case as predicted since the condition of the saturation level is not fulfilled.

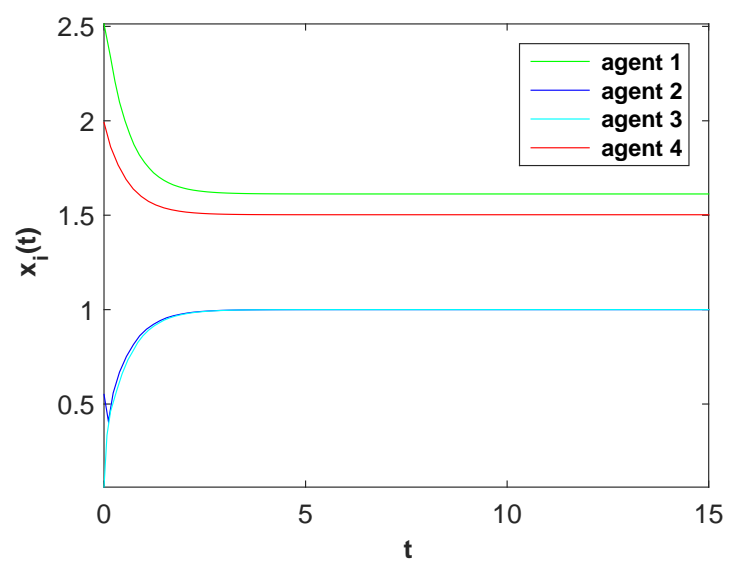

Fig. 4: The state evolution under static triggering law (8).

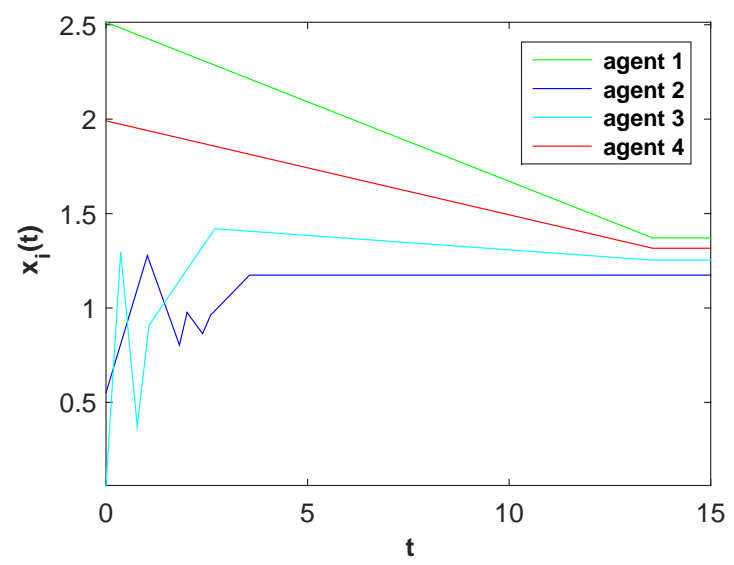

Fig. 5: The state evolution under dynamic triggering law (12).

\section{Conclusions}

In this paper, we proposed a static and a dynamic eventtriggered control law for multi-agent systems subject to output saturation. We showed that, if the communication graph is undirected and connected, both event-triggered control laws solve the consensus problem if and only if the average of the initial states is within the saturation level. In addition, the dynamic event-triggered control law was shown to be free of Zeno behavior. Future research directions include considering directed communication graphs, input saturations and interconnection saturations. 


\section{References}

[1] R. Olfati-Saber and R. M. Murray, "Consensus problems in networks of agents with switching topology and time-delays," IEEE Transactions on Automatic Control, vol. 49, no. 9, pp. 1520-1533, 2004.

[2] W. Ren, R. W. Beard, and E. M. Atkins, "Information consensus in multivehicle cooperative control," IEEE Control Systems Magazine, vol. 27, no. 2, pp. 71-82, 2007.

[3] B. Liu, W. Lu, and T. Chen, "Consensus in networks of multiagents with switching topologies modeled as adapted stochastic processes," SIAM Journal on Control and Optimization, vol. 49, no. 1, pp. 227-253, 2011.

[4] K. You and L. Xie, "Network topology and communication data rate for consensusability of discrete-time multi-agent systems," IEEE Transactions on Automatic Control, vol. 56, no. 10, pp. 2262-2275, 2011.

[5] T. Yang, Z. Meng, D. V. Dimarogonas, and K. H. Johansson, "Global consensus for discrete-time multi-agent systems with input saturation constraints," Automatica, vol. 50, no. 2, pp. 499-506, 2014.

[6] Z. Meng, Z. Zhao, and Z. Lin, "On global leader-following consensus of identical linear dynamic systems subject to actuator saturation," Systems \& Control Letters, vol. 62, no. 2, pp. 132-142, 2013.

[7] Y.-H. Lim and H.-S. Ahn, "Consensus with output saturations," arXiv preprint arXiv:1606.05980, 2016.

[8] Q. Wang and C. Sun, "Conditions for consensus in directed networks of agents with heterogeneous output saturation," IET Control Theory \& Applications, vol. 10, no. 16, pp. 21192127, 2016.

[9] K. J. Åström and B. Bernhardsson, "Comparison of periodic and event based sampling for first-order stochastic systems," in Proceedings of the 14th IFAC World congress, vol. 11. Citeseer, 1999, pp. 301-306.

[10] P. Tabuada, "Event-triggered real-time scheduling of stabilizing control tasks," IEEE Transactions on Automatic Control, vol. 52, no. 9, pp. 1680-1685, 2007.

[11] X. Wang and M. D. Lemmon, "Event-triggering in distributed networked control systems," IEEE Transactions on Automatic Control, vol. 56, no. 3, pp. 586-601, 2011.

[12] W. Heemels, K. H. Johansson, and P. Tabuada, "An introduction to event-triggered and self-triggered control," in Decision and Control (CDC), 2012 IEEE 51st Annual Conference on. IEEE, 2012, pp. 3270-3285.

[13] D. V. Dimarogonas, E. Frazzoli, and K. H. Johansson, "Distributed event-triggered control for multi-agent systems," IEEE Transactions on Automatic Control, vol. 57, no. 5, pp. 1291-1297, 2012.

[14] E. Garcia, Y. Cao, H. Yu, P. Antsaklis, and D. Casbeer, "Decentralised event-triggered cooperative control with limited communication," International Journal of Control, vol. 86, no. 9, pp. 1479-1488, 2013.

[15] G. S. Seyboth, D. V. Dimarogonas, and K. H. Johansson, "Event-based broadcasting for multi-agent average consensus," Automatica, vol. 49, no. 1, pp. 245-252, 2013.

[16] X. Meng, L. Xie, Y. C. Soh, C. Nowzari, and G. J. Pappas, "Periodic event-triggered average consensus over directed graphs," in Decision and Control (CDC), 2015 IEEE 54th Annual Conference on. IEEE, 2015, pp. 4151-4156.

[17] C. Nowzari and J. Cortés, "Distributed event-triggered coordination for average consensus on weight-balanced digraphs," Automatica, vol. 68, pp. 237-244, 2016.

[18] X. Meng and T. Chen, "Event based agreement protocols for multi-agent networks," Automatica, vol. 49, no. 7, pp. 2125$2132,2013$.
[19] Y. Fan, G. Feng, Y. Wang, and C. Song, "Distributed eventtriggered control of multi-agent systems with combinational measurements," Automatica, vol. 49, no. 2, pp. 671-675, 2013.

[20] X. Yi, W. Lu, and T. Chen, "Distributed event-triggered consensus for multi-agent systems with directed topologies," in Proceedings of the 2016 Chinese Control and Decision Conference, 2016, pp. 807-813.

[21] X. Yi, J. Wei, D. V. Dimarogonas, and K. H. Johansson, "Formation control for multi-agent systems with connectivity preservation and event-triggered controllers," arXiv:1611.03105, 2016.

[22] X. Yi, W. Lu, and T. Chen, "Pull-based distributed eventtriggered consensus for multiagent systems with directed topologies," IEEE Transactions on Neural Networks and Learning Systems, vol. 28, no. 1, pp. 71-79, Jan 2017.

[23] K. H. Johansson, M. Egerstedt, J. Lygeros, and S. Sastry, "On the regularization of Zeno hybrid automata," Systems \& Control Letters, vol. 38, no. 3, pp. 141-150, 1999.

[24] A. Girard, "Dynamic triggering mechanisms for eventtriggered control," IEEE Transactions on Automatic Control, vol. 60, no. 7, pp. 1992-1997, 2015.

[25] M. Mesbahi and M. Egerstedt, Graph Theoretic Methods in Multiagent Networks. Princeton University Press, 2010.

[26] H. K. Khalil, Nonlinear systems, 3rd. Prentice-Hall, New Jersey, 2002. 